\title{
Connection between sinc approximation for high-energy absorption cross section and shadow of black holes
}

\author{
Ali Övgün* \\ Physics Department, Eastern Mediterranean University, \\ Famagusta, 99628 North Cyprus via Mersin 10, Turkey.
}

(Dated: August 17, 2021)

\begin{abstract}
This letter aims to show the connection between the sinc approximation for high-energy absorption cross section and the shadow radius of the spherically symmetric black hole. This connection can give a physical interpretation of the absorption cross section in the eikonal limit parameters. Moreover, the use of this alternative way, one can extract its shadow radius from the absorption cross section in high energy limits to gain more information about the black hole spacetime. Our results indicate that the increasing the value of the shadow radius of the black hole, exponentially increase the the absorption cross section of the black hole in high-energy limits which can be captured by the Event Horizon Telescope (EHT) collaboration.
\end{abstract}

PACS numbers: 04.70.Bw, 95.30.Sf, 97.60.Lf, 98.62.Sb

Keywords: Gravitation; Black hole; Shadow; High-energy physics; Cross-section; Null geodesics

\section{INTRODUCTION}

Black holes are some of the most mysterious objects in the universe which are a region of space within which the force of gravity is too strong that nothing can escape, not even light. In 2015 the Laser Interferometer Gravitational wave Observatory (LIGO) [1] detector detected gravitational waves from a merger of two stellar-mass black holes, soon after the Event Horizon Telescope (EHT) collaboration made headlines in 2019 by capturing the first direct image of a black hole at the center of a Messier 87 (M87*) galaxy [2]. These observational evidences not only prove the success of general relativity, but also open new gates in understanding black hole physics.

The intense gravity of a black hole curves the spacetime geometry, similarly with magnifying glass, and forming the larger image of the shadow cast. Photons are trapped by the black hole that cross event horizon and cast a shadow on its bright surrounding emission from hot infalling gas [3, 4]. There are many bright photon rings around this shadow which are formed by strong gravity. To detect the properties of black hole, one should look at its photon rings which are the fingerprint of black hole. This apparent boundary is also called critical curve where the light rays will asymptotically approach a bound photon orbit. That's why the photons near the critical curve orbit the black hole too many times [5-7].

Black holes are ever so slightly gray with emitting radiation like a black body with finite temperature, known as Hawking temperature, after the physicist Stephen Hawking who proposed it using the quantum field theory around the event horizon of the black hole [8]. Consequently, this effective idea of absorption and emission of particles in the strong gravity region gets physicist attentions [10-15]. There are stimulating phenomena between the interactions of fields and black holes such as scattering [16, 17], emission [18-24] and absorption [25-38].

To completely understand the spacetime properties, fundamental aspects of classical and quantum physics should be understood in high energy levels. At high energy physics, it is well-known that the absorption cross section is a measure for the probability of an absorption process and the importance of the absorption and scattering of fields are their relation of the fields around the black hole, gravitational waves and active galactic nuclei $[39,40]$. Sanchez show that the absorption cross section oscillated around the constant geometric-optics value for a black hole, which is related with the photon sphere, on the other hand, it increases monotonically with increasing frequency for ordinary material sphere [26]. Hence, one can easily understand the difference between them as well as between the different types of black holes. The photon sphere, which is a hypersurface of unstable null circular geodesics, can be seen as a capture cross section of the black hole because it is located at the maximum of the effective potential as well as at critical impact parameter $b_{c}$, coming null right rays from infinity reaching the photon sphere by circling. Hence the cross section of the photon sphere is linked with critical impact parameter and one can limit the value of absorption cross section to see that it is a characteristic property of the black holes at low energies (where the cross section equals to black hole area) $[15,41]$ and also it is shown at high energies [42] using the geometrical cross section of the photon sphere via method of null geodesics and wave theories [10, 43].

The work of Decanini et al. [42] simply show that the fluctuations around the limiting value in terms of black holes and it is used the Regge pole techniques to prove that the oscillatory pattern of the absorption cross section related with a $\operatorname{sinc}(\mathrm{x})$ function included photon sphere. In the sinc approximation, the absorption cross-section equals to the geomet- 
ric cross-section and oscillatory part of the absorption crosssection. The correspondence between the high-energy absorption cross section at limiting values and strong deflection angle are given in [44-46]. The relation between the quasinormal modes (QNM), which are the characteristic 'sound' of black holes and neutron stars, and null geodesics quantities are shown by Cardoso et al. in [47-49]. Moreover, the correspondence between the strong gravitational lensing and quasinormal modes are given by Stefanov et al. in [50]. On the other hand, Wei et al. show that there is a connection between the angular velocity with the radius of the shadow in [51]. Moreover, the corresponding between the black hole shadow and the energy emission rate of the black hole is given by Wei et al. [52] as:

$$
\frac{d^{2} E(\omega)}{d \omega d t}=\frac{2 \pi^{2} \pi R_{s}^{2}}{e^{\omega / T}-1} \omega^{3},
$$

where the $R_{s}$ stands for the shadow radius of the black hole, $\omega$ is the photon frequency and $T$ is for Hawking temperature of the black hole. However, as far as we know, no work has shown how the sinc approximation for high-energy absorption cross section related with the shadow radius of the black hole.

The main aim of the work is to show the connection between the sinc approximation for high-energy absorption cross section and the shadow radius of the black hole.

This letter is organized as follows: in section 2 we show the correspondence between the high-energy absorption cross section and shadow radius of the black hole, and then we give an example. The last section 3 is devoted to conclusions.

\section{CORRESPONDENCE BETWEEN HIGH-ENERGY ABSORPTION CROSS SECTION AND SHADOW RADIUS OF BLACK HOLES}

The line element which represents a static and spherically symmetric black hole is

$$
d s^{2}=-A(r) d t^{2}+\frac{d r^{2}}{A(r)}+r^{2}\left(d \theta^{2}+\sin ^{2} \theta d \phi^{2}\right),
$$

where the $A(r)$ is the metric function that only depends on the radial coordinate $r$. To analyse the shadow of the black hole, we use the standard Hamilton-Jacobi approach for a photon. The equatorial plane $\theta=\frac{\pi}{2}$ should be considered due to the spherical symmetry. Two constants of motion, energy $E=-p_{t}$ and angular momentum $L=p_{\phi}$, (where the $p_{\mu}$ is the photon momenta) are obtained using the Hamiltonian of the spacetime:

$$
H=\frac{1}{2} g^{\mu v} p_{\mu} p_{v}=\frac{1}{2}\left(\frac{L^{2}}{r^{2}}-\frac{E^{2}}{A(r)}+\frac{\dot{r}^{2}}{A(r)}\right)=0,
$$

where $\dot{r}=\partial H / \partial p_{r}$. Using the (3), the null geodesic equation of the light ray can be calculated, and the effective poten- tial $V$ which depends on the radial motion direction only is given by

$$
V+\dot{r}^{2}=0, \quad V=A(r)\left(\frac{L^{2}}{r^{2}}-\frac{E^{2}}{A(r)}\right) .
$$

The stable/unstable circular orbit requires $V^{\prime \prime}(r)>0$ which admits a minimum/maximum of the effective potential. Then the circular geodesics satisfy [3-5].

$$
\left.V(r)\right|_{r=r_{c}}=0,\left.\quad V^{\prime}(r)\right|_{r=r_{c}}=0,
$$

where the critical impact parameter $b_{c} \equiv \frac{L}{E}=\frac{r_{c}}{\sqrt{A\left(r_{c}\right)}}$ and $r_{c}$ is the radius of the photon sphere, which can be calculated by finding the largest root of the this relation:

$$
\frac{A^{\prime}\left(r_{c}\right)}{A\left(r_{c}\right)}=\frac{2}{r_{c}},
$$

As observed from the distant static observer at the position $r_{0}$, the radius of the black hole shadow is [53]

$$
R_{s}=r_{c} \sqrt{\frac{A\left(r_{0}\right)}{A\left(r_{c}\right)}},
$$

and for large distant observer $\left(A\left(r_{0}\right)=1\right)$, it reduces to

$$
R_{s}=\frac{r_{c}}{\sqrt{A\left(r_{c}\right)}}
$$

At low-energy limits, the calculation of the absorption cross-section (equals to black hole horizon area) was done for spherically symmetric black holes by Higuchi [41]. Moreover, at the high-energy limits, using the null geodesics, the absorption cross-section is related with the classical capture cross-section (known as geometric cross-section) and authors show that it is possible to write absorption cross-section in term of photon sphere at eikonal limit (high-energy limit). $\sigma_{\text {geo }}=\pi b_{c}^{2}[26,42]$.

Here we use the sinc approximation to show its connection with the radius of the shadow of the black hole. To do so, in the sinc approximation, we first write the high-energy absorption cross section for a black hole spacetime [42]:

$$
\sigma_{\mathrm{abs}}(\omega) \approx \sigma_{\mathrm{lim}}+\sigma_{\mathrm{osc}},
$$

where the absorption cross section $\sigma_{\mathrm{abs}}$ at $\omega \rightarrow \infty$ defined as:

$$
\sigma_{\lim }=\sigma_{\mathrm{geo}}=\frac{\pi b_{\mathrm{c}}^{2}}{\Gamma(2)},
$$

and the oscillating part $\sigma_{\text {osc }}$ given by

$$
\sigma_{\mathrm{osc}}=-8 \pi \eta_{\mathrm{c}} e^{-\pi \eta_{\mathrm{c}}} \operatorname{sinc}\left(\omega T_{\mathrm{c}}\right) \sigma_{\mathrm{geo}}
$$


in which $\operatorname{sinc}(x) \equiv \frac{\sin x}{x}$, the orbital period $T_{\mathrm{c}}=2 \pi b_{\mathrm{c}}$ and $\sigma_{\text {geo }}$ is the geometrical cross section. On the other hand, the critical impact parameter and other parameter are given by:

$$
b_{\mathrm{c}}=\frac{r_{\mathrm{c}}}{\sqrt{A\left(r_{c}\right)}}, \quad \eta_{\mathrm{c}}=\sqrt{A\left(r_{c}\right)-\frac{1}{2} r_{\mathrm{c}}^{2} A\left(r_{c}\right)^{\prime \prime}} .
$$

It is noted that the high-energy absorption cross section $\sigma_{\text {abs }}$ only depends on the parameters $b_{\mathrm{c}}$ and $\eta_{\mathrm{c}}$. Then the absorption cross section in the eikonal limit is defined by:

$$
\sigma_{o s c}=-4 \pi \frac{\lambda b_{c}^{2}}{w} e^{-\pi \lambda b_{c}} \sin \left(2 \pi w b_{c}\right),
$$

where $\lambda$ is the Lyapunov exponent[47]:

$$
\lambda^{2}=\frac{f\left(r_{c}\right)}{2 r_{c}^{2}}\left[2 f\left(r_{c}\right)-r_{c}^{2} f^{\prime \prime}\left(r_{c}\right)\right]
$$

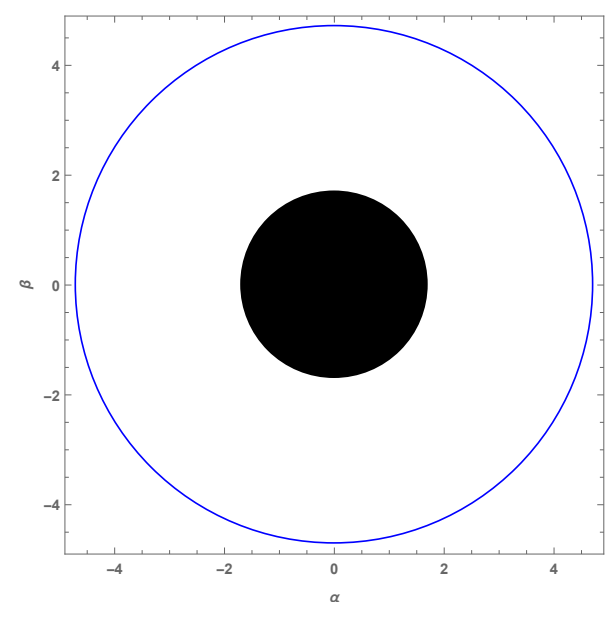

FIG. 1. The shadow of the charged black hole for the value of $M=1$ and $Q=0.5$.

To show the connection between the sinc approximation of high-energy cross section and shadow radius of the black hole, the parameters of the absorption cross-section are needed to be written in terms of the radius of the shadow which is related to critical impact parameter of the black hole. By this way, using the Eq.s (8)-(9) the simple relations for the sinc approximation of high-energy absorption cross section with the shadow radius of the black hole is derived as follows

$$
\sigma_{\mathrm{abs}}(\omega)=-8 \pi \eta_{\mathrm{c}} e^{-\pi \eta_{\mathrm{c}}} \operatorname{sinc}\left(\omega 2 \pi R_{s}\right) \pi R_{s}^{2}+\pi R_{s}^{2}
$$

Here, we have established the relation between the absorption cross section and the shadow radius of black holes.

Now we can give a example for this relation. The metric function of the charged black hole is given by

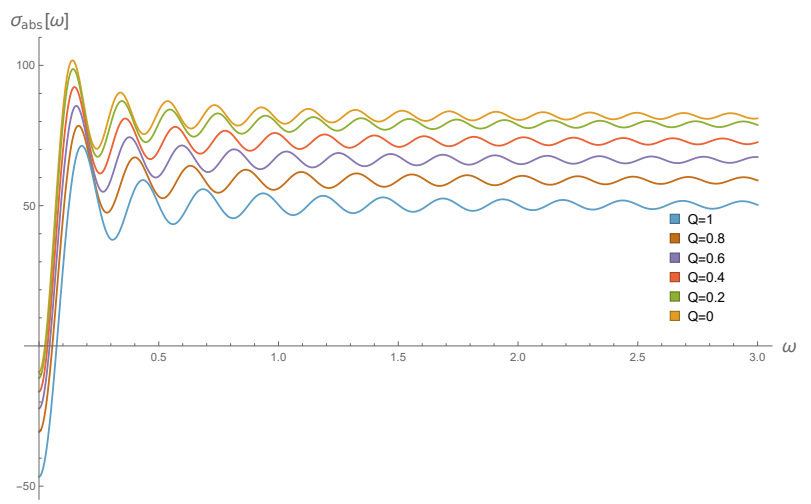

FIG. 2. Total absorption cross section of the charged black hole using the sinc approximation.

$$
A(r)=1-\frac{2 M}{r}+\frac{Q}{r^{2}},
$$

where $M$ is the mass of the black hole, and $Q$ is the charge of the black hole (for the charge of Reissner-Nordström $q=$ $\sqrt{Q})$.

The event horizon of the charged black hole is located at: $r \rightarrow M+\sqrt{M^{2}-Q}$. Moreover, using the Eq. 6 the photon sphere of the charged black hole $r_{c}$ is calculated as

$$
r_{c}=\frac{1}{2} \sqrt{9 M^{2}-8 Q}+\frac{3 M}{2},
$$

and using the Eq. 8 the shadow radius of the charged black hole is obtained as

$$
R_{s}=\frac{\sqrt{9 M^{2}-8 Q}+3 M}{\sqrt{\frac{M\left(\sqrt{9 M^{2}-8 Q}-3 M\right)}{2 Q}+2}}
$$

where is plotted in Fig. 1. On the other hand total absorption cross section via sinc approximation using the Eq. 9 for the charged black hole is plotted in Fig. 2 which shows that high-energy absorption cross section in the sinc limit is varying with the changing the value of the charge parameter. On the other hand, it can be seen that the increasing the charge parameter, decreases the high-energy absorption cross-section in the sinc approximation.

Afterwards, we use the Eq. 18 in the Eq. 15 to derive the sinc approximation equation of the high-energy absorption cross section in the parameter of the shadow radius of the charged black hole and plot it in Fig. 3, 4, and 5, which show that in the eikonal limits, increasing the value of the shadow radius of the black hole, varies the high-energy absorption cross-section in the sinc approximation. For the small value of the shadow radius of the black hole, the high-energy absorption cross-section in the sinc approximation increases slowly 
by fluctuated. However, for the large value of the shadow radius of the black hole, the high-energy absorption crosssection in the sinc approximation exponentially increases.

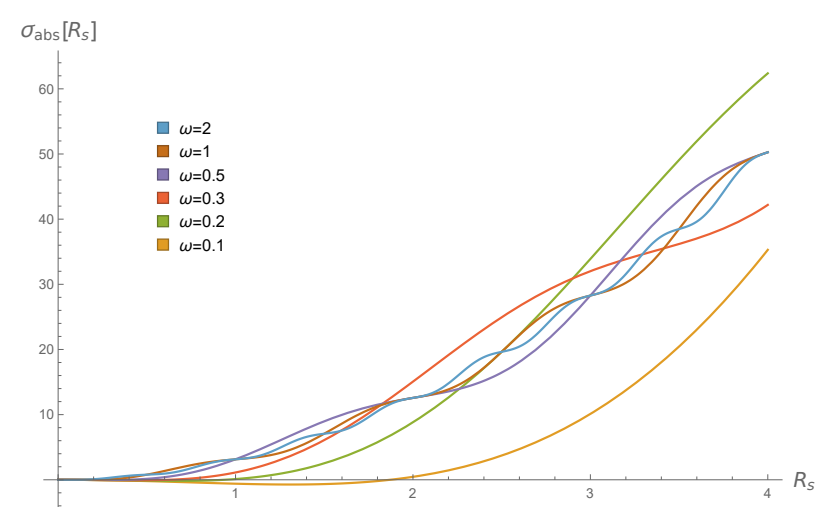

FIG. 3. The relation between the total absorption cross section via sinc approximation and the small shadow radius of the black hole for $R_{s}<4$.

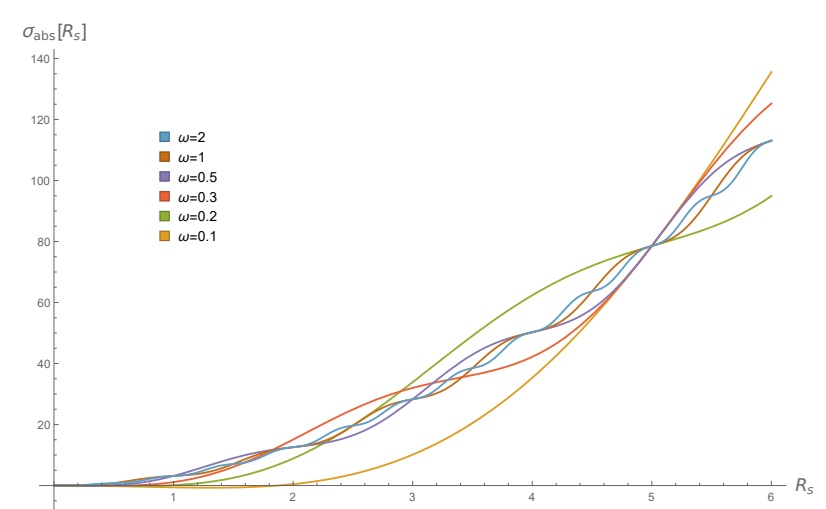

FIG. 4. The relation between the total absorption cross section via sinc approximation and the shadow radius of the black hole for $R_{s}<$ 6.

\section{CONCLUSION}

In this letter, we have shown that the sinc approximation for high-energy absorption cross section in eikonal limits is connected with the shadow radius of the spherically symmetric black hole. To do so, we calculate the null geodesics and obtain the critical values of the impact parameter to find the shadow radius of the black hole. Then, by using the Regge pole technique, the compact form of the high-energy absorption cross section in eikonal limit, known as sinc approximation is obtained. Then the correspondence between the shadow radius and the sinc approximation for the high-energy absorption cross section is shown explicitly. Although we have focused our analysis in the cases of charged black hole,

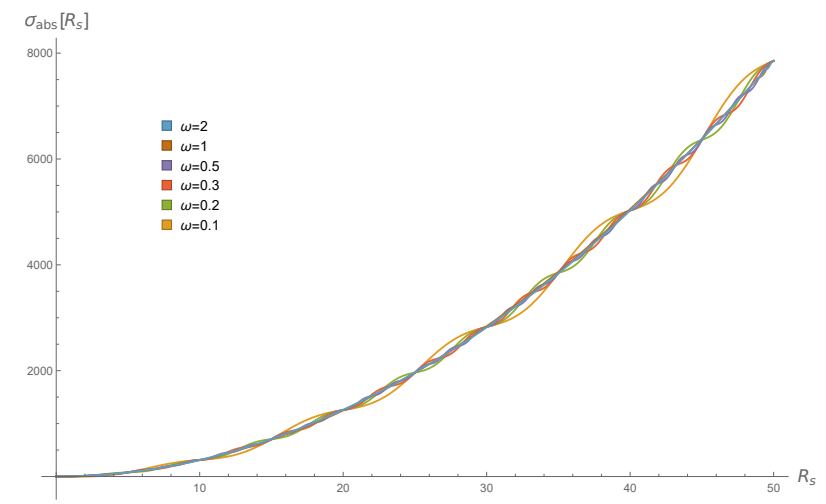

FIG. 5. The relation between the total absorption cross section via sinc approximation and the large shadow radius of the black hole for $R_{s}<50$.

this method can be directly generalized to any other black hole solutions.

Finally, the results show that if the value of the shadow radius of the black hole is increased, the sinc approximation of high-energy cross section raises. For the small value of the shadow radius of the black hole, the high-energy absorption cross-section in the sinc approximation increases slowly by fluctuated. However, for the large value of the shadow radius of the black hole, the high-energy absorption cross-section in the sinc approximation exponentially increases.

The connection of the sinc approximation for high-energy absorption cross section with the radius of the rotating black holes may reveal more information compared to the present case which can captured by the EHT collaboration. This is the next stage of our investigation that interests us.

*ali.ovgun@emu.edu.tr

[1] B. P. Abbott et al. [LIGO Scientific and Virgo], "Observation of Gravitational Waves from a Binary Black Hole Merger," Phys. Rev. Lett. 116, no.6, 061102 (2016)

[2] K. Akiyama et al. [Event Horizon Telescope], "First M87 Event Horizon Telescope Results. I. The Shadow of the Supermassive Black Hole," Astrophys. J. Lett. 875, L1 (2019)

[3] H. Falcke, F. Melia and E. Agol, "Viewing the shadow of the black hole at the galactic center," Astrophys. J. Lett. 528, L13 (2000)

[4] V. Perlick and O. Y. Tsupko, "Calculating black hole shadows: review of analytical studies,"

[5] A. Övgün and İ. Sakallı, "Testing generalized Einstein-Cartan-Kibble-Sciama gravity using weak deflection angle and shadow cast," Class. Quant. Grav. 37, no.22, 225003 (2020)

[6] A. Övgün, "Black hole with confining electric potential in 
scalar-tensor description of regularized 4-dimensional EinsteinGauss-Bonnet gravity,” Phys. Lett. B 820, 136517 (2021)

[7] A. Övgün, İ. Sakallı and J. Saavedra, "Shadow cast and Deflection angle of Kerr-Newman-Kasuya spacetime," JCAP 10, 041 (2018)

[8] S. W. Hawking, "Black hole explosions," Nature 248, 30-31 (1974).

[9] R. A. Matzner, "Scattering of Massless Scalar Waves by a Schwarzschild Singularity,” J. Math. Phys. 9, 163 (1968).

[10] B. Mashhoon, "Scattering of Electromagnetic Radiation from a Black Hole,” Phys. Rev. D 7, 2807-2814 (1973)

[11] A. A. Starobinsky, "Amplification of waves reflected from a rotating "black hole".," Sov. Phys. JETP 37, no.1, 28-32 (1973).

[12] R. Fabbri, "Scattering and absorption of electromagnetic waves by a Schwarzschild black hole," Phys. Rev. D 12, 933-942 (1975).

[13] D. N. Page, "Particle Emission Rates from a Black Hole: Massless Particles from an Uncharged, Nonrotating Hole," Phys. Rev. D 13, 198-206 (1976). .

[14] W. G. Unruh, "Absorption Cross-Section of Small Black Holes,” Phys. Rev. D 14, 3251-3259 (1976).

[15] S. R. Das, G. W. Gibbons and S. D. Mathur, "Universality of low-energy absorption cross-sections for black holes," Phys. Rev. Lett. 78, 417-419 (1997).

[16] S. Dolan, C. Doran and A. Lasenby, "Fermion scattering by a Schwarzschild black hole,” Phys. Rev. D 74, 064005 (2006)

[17] L. C. B. Crispino, S. R. Dolan, A. Higuchi and E. S. de Oliveira, "Inferring black hole charge from backscattered electromagnetic radiation,” Phys. Rev. D 90, no.6, 064027 (2014)

[18] D. Singleton and S. Wilburn, "Hawking radiation, Unruh radiation and the equivalence principle," Phys. Rev. Lett. 107, 081102 (2011)

[19] L. C. B. Crispino, "Synchrotron scalar radiation from a source in ultrarelativistic circular orbits around a Schwarzschild black hole," Phys. Rev. D 77, 047503 (2008)

[20] A. Övgün and K. Jusufi, "Massive vector particles tunneling from noncommutative charged black holes and their GUPcorrected thermodynamics," Eur. Phys. J. Plus 131, no.5, 177 (2016)

[21] L. A. Oliveira, L. C. B. Crispino and A. Higuchi, "Scalar radiation from a radially infalling source into a Schwarzschild black hole in the framework of quantum field theory," Eur. Phys. J. C 78, no.2, 133 (2018)

[22] S. K. Modak and D. Singleton, "Baryogenesis via Hawking-like Radiation in the FRW Space-time,” Eur. Phys. J. C 75, no.5, 200 (2015)

[23] E. T. Akhmedov, V. Akhmedova and D. Singleton, "Hawking temperature in the tunneling picture," Phys. Lett. B 642, 124128 (2006)
[24] I. Sakalli and A. Ovgun, "Hawking Radiation and Deflection of Light from Rindler Modified Schwarzschild Black Hole,’ EPL 118, no.6, 60006 (2017)

[25] T. Nakamura and H. Sato, "Absorption of Massive Scalar Field by a Charged Black Hole," Phys. Lett. B 61, 371-374 (1976)

[26] N. G. Sanchez, "Absorption and Emission Spectra of a Schwarzschild Black Hole,” Phys. Rev. D 18, 1030 (1978).

[27] M. A. A. Paula, L. C. S. Leite and L. C. B. Crispino, "Electrically charged black holes in linear and nonlinear electrodynamics: Geodesic analysis and scalar absorption," Phys. Rev. D 102, no.10, 104033 (2020)

[28] H. C. D. Lima, C. L. Benone and L. C. B. Crispino, "Scalar absorption: Black holes versus wormholes,” Phys. Rev. D 101, no.12, 124009 (2020)

[29] R. B. Magalhães, L. C. S. Leite and L. C. B. Crispino, "Schwarzschild-like black holes: Light-like trajectories and massless scalar absorption,” Eur. Phys. J. C 80, no.5, 386 (2020)

[30] R. B. Magalhães, L. C. S. Leite and L. C. B. Crispino, "Absorption by deformed black holes," Phys. Lett. B 805, 135418 (2020)

[31] C. L. Benone, E. S. de Oliveira, S. R. Dolan and L. C. B. Crispino, "Absorption of a massive scalar field by a charged black hole,” Phys. Rev. D 89, no.10, 104053 (2014)

[32] C. L. Benone and L. C. B. Crispino, "Massive and charged scalar field in Kerr-Newman spacetime: Absorption and superradiance," Phys. Rev. D 99, no.4, 044009 (2019)

[33] C. F. B. Macedo and L. C. B. Crispino, "Absorption of planar massless scalar waves by Bardeen regular black holes," Phys. Rev. D 90, no.6, 064001 (2014)

[34] C. L. Benone, L. C. S. Leite, L. C. B. Crispino and S. R. Dolan, "On-axis scalar absorption cross section of Kerr-Newman black holes: Geodesic analysis, sinc and low-frequency approximations,” Int. J. Mod. Phys. D 27, no.11, 1843012 (2018)

[35] A. Delhom, C. F. B. Macedo, G. J. Olmo and L. C. B. Crispino, "Absorption by black hole remnants in metric-affine gravity," Phys. Rev. D 100, no.2, 024016 (2019)

[36] H. Huang, J. Chen, Y. Wang and Y. Jin, "Absorption of a Massive Scalar Field by Wormhole Space-Times," Int. J. Theor. Phys. 56, no.4, 1150-1158 (2017)

[37] L. C. B. Crispino, A. Higuchi and G. E. A. Matsas, "Lowfrequency absorption cross section of the electromagnetic waves for the extreme Reissner-Nordstrom black holes in higher dimensions," Phys. Rev. D 82, 124038 (2010)

[38] L. C. B. Crispino and E. S. Oliveira, "Electromagnetic absorption cross section of Reissner-Nordstrom black holes," Phys. Rev. D 78, 024011 (2008)

[39] L. Ferrarese and H. Ford, "Supermassive black holes in galactic nuclei: Past, present and future research," Space Sci. Rev. 116, 523-624 (2005) 
[40] A. Marconi, G. Risaliti, R. Gilli, L. K. Hunt, R. Maiolino and M. Salvati, "Local supermassive black holes, relics of active galactic nuclei and the x-ray background," Mon. Not. Roy. Astron. Soc. 351, 169 (2004)

[41] A. Higuchi, "Low frequency scalar absorption cross-sections for stationary black holes," Class. Quant. Grav. 18, L139 (2001)

[42] Y. Decanini, G. Esposito-Farese and A. Folacci, "Universality of high-energy absorption cross sections for black holes," Phys. Rev. D 83, 044032 (2011)

[43] C. W. Misner, K. S. Thorne and J. A. Wheeler, "Gravitation," Gravitation (W. H. Freeman and Company, San Francisco, 1973)

[44] V. Bozza, "Gravitational Lensing by Black Holes," Gen. Rel. Grav. 42, 2269-2300 (2010).

[45] K. S. Virbhadra and G. F. R. Ellis, "Gravitational lensing by naked singularities,” Phys. Rev. D 65, 103004 (2002)

[46] C. M. Claudel, K. S. Virbhadra and G. F. R. Ellis, "The Geometry of photon surfaces," J. Math. Phys. 42, 818-838 (2001)

[47] V. Cardoso, A. S. Miranda, E. Berti, H. Witek and
V. T. Zanchin, "Geodesic stability, Lyapunov exponents and quasinormal modes," Phys. Rev. D 79, 064016 (2009)

[48] V. Cardoso, J. L. Costa, K. Destounis, P. Hintz and A. Jansen, "Quasinormal modes and Strong Cosmic Censorship," Phys. Rev. Lett. 120, no.3, 031103 (2018)

[49] V. Cardoso and P. Pani, "The observational evidence for horizons: from echoes to precision gravitational-wave physics," [arXiv:1707.03021 [gr-qc]].

[50] I. Z. Stefanov, S. S. Yazadjiev and G. G. Gyulchev, "Connection between Black-Hole Quasinormal Modes and Lensing in the Strong Deflection Limit," Phys. Rev. Lett. 104, 251103 (2010)

[51] S. W. Wei and Y. X. Liu, "Null Geodesics, Quasinormal Modes, and Thermodynamic Phase Transition for Charged Black Holes in Asymptotically Flat and dS Spacetimes," Chin. Phys. C 44, no.11, 115103 (2020).

[52] S. W. Wei and Y. X. Liu, "Observing the shadow of EinsteinMaxwell-Dilaton-Axion black hole," JCAP 11, 063 (2013).

[53] R. A. Konoplya, "Quantum corrected black holes: quasinormal modes, scattering, shadows,” Phys. Lett. B 804, 135363 (2020) 